\title{
Quantitative SERS Assay on a Single Chip Enabled by Electrochemically Assisted Regeneration: A Method for Detection of Melamine in Milk
}

Viehrig, Marlitt; Rajendran, Sriram T.; Sanger, Kuldeep; Schmidt, Michael S.; Alstrøm, Tommy S.; Rindzevicius, Tomas; Zor, Kinga; Boisen, Anja

Published in:

Analytical Chemistry

Link to article, DOI:

10.1021/acs.analchem.9b05060

Publication date:

2020

Document Version

Peer reviewed version

Link back to DTU Orbit

Citation (APA):

Viehrig, M., Rajendran, S. T., Sanger, K., Schmidt, M. S., Alstrøm, T. S., Rindzevicius, T., Zor, K., \& Boisen, A. (2020). Quantitative SERS Assay on a Single Chip Enabled by Electrochemically Assisted Regeneration: A Method for Detection of Melamine in Milk. Analytical Chemistry, 92(6), 4317-4325.

https://doi.org/10.1021/acs.analchem.9b05060

\section{General rights}

Copyright and moral rights for the publications made accessible in the public portal are retained by the authors and/or other copyright owners and it is a condition of accessing publications that users recognise and abide by the legal requirements associated with these rights.

- Users may download and print one copy of any publication from the public portal for the purpose of private study or research.

- You may not further distribute the material or use it for any profit-making activity or commercial gain

- You may freely distribute the URL identifying the publication in the public portal 


\title{
Quantitative SERS assay on a single chip enabled by
}

\section{electrochemically assisted regeneration: a method for}

\section{detection of melamine in milk}

\author{
Marlitt Viehrig 1, $^{*}$, Sriram T. Rajendran ${ }^{1}$, Kuldeep Sanger ${ }^{1}$, Michael S. Schmidt ${ }^{1}$, \\ Tommy S. Alstrøm ${ }^{1,2}$, Tomas Rindzevicius ${ }^{1}$, Kinga Zór ${ }^{1,{ }^{*}}$ and Anja Boisen ${ }^{1}$
}

${ }^{1}$ The Danish National Research Foundation and Villum Foundation's Center for Intelligent Drug Delivery and Sensing Using Microcontainers and Nanomechanics (IDUN), Department of Health Technology, Technical University of Denmark, Kgs. Lyngby, 2800, Denmark.

${ }^{2}$ Department of Applied Mathematics and Computer Science, Technical University of Denmark, Kgs. Lyngby, 2800, Denmark.

E-mail: mvie@dtu.dk, kinzo@dtu.dk 


\begin{abstract}
Reusability of sensors is relevant when aiming to decrease variation between measurements, cost and time of analysis. We present an electrochemically assisted surface-enhanced Raman spectroscopy (SERS) platform with the capability to reverse the analyte-surface interaction, without damaging the SERS substrate, allowing for efficient sensor reuse. The platform was used in combination with a sample pre-treatment step, when detecting melamine from milk. We found that the electrochemically enhanced analyte-surface interaction results in significant improvement in detection sensitivity, with detection limits $(0.01 \mathrm{ppm}$ in PBS and $0.3 \mathrm{ppm}$ in milk) below the maximum limit allowed in food samples. The reversibility of interaction enabled continuous measurement in aqueous solution and a complete quantitative assay on a single SERS substrate.
\end{abstract}

Keywords: EC-SERS, SERS, Gold-capped (Au-capped) Si nanopillars, Reversibility, Reusability Quantification, Melamine, Milk 


\section{Introduction}

In recent years, surface-enhanced Raman spectroscopy (SERS) based sensing has become relevant in various scientific and technological fields, including analytical chemistry and biology, ${ }^{1}$ biomedical diagnostics, ${ }^{2,3}$ forensics science, ${ }^{4,5}$ drug discovery, ${ }^{6}$ environmental monitoring ${ }^{7}$ and food safety. ${ }^{8}$ The main advantages of SERS are the specificity of the recorded signal, and fast analysis. ${ }^{9}$ Additionally, it can be combined with miniaturized laboratory concepts for on-site, point of need or point of care detection, such as lab-on-a-disc ${ }^{10-13}$ and lab-on-a-chip systems. ${ }^{14}$ The potential of the technology is further supported by the availability of high quality commercial substrates ${ }^{15-17}$ as well as by the access to miniaturized, portable Raman systems. ${ }^{18-24}$

However, despite the extensive research and development efforts, SERS has not yet made the leap to become a standard analytical tool. The primary challenges of SERS-based sensing technologies are related to reproducible, sensitive, affordable ${ }^{25,26}$ quantification. ${ }^{9,27-29}$ One of the limitation of the technology is the lack of possibilities for real-time, in-line monitoring, a crucial aspect when aiming for quality control. To enable reliable quantification, several approaches have been proposed, which provide uniform, homogeneous substrates and consequently reproducible sensors. ${ }^{9,30-32}$ Sensitivity can be improved by means of specific surface functionalization facilitating the localization of analytes in close proximity of the electromagnetic (EM) hotspots, ${ }^{30,33,34}$ where the EM-field enhancement is highest. ${ }^{35}$ Additionally, when combining electrochemistry with SERS and utilizing the SERS substrate as working electrode, a precise control of surface charges can be achieved. This effect has been shown to improve the detection limits of SERS sensors, when measuring, for example, thiols, ${ }^{36}$ uric acid as a preeclampsia marker, ${ }^{37,38}$ drug metabolites, ${ }^{39}$ antibiotics, ${ }^{40}$ screening and identification of bacterial strains ${ }^{41}$ as well as detection of the milk adulterant melamine. ${ }^{42}$

In a quantitative SERS assay usually multiple SERS chips are used for calibration and detection of the unknown sample, which relies on acceptable chip-to-chip reproducibility in the SERS signal 
intensity. ${ }^{43}$ A state-of-the-art inter-chip variation in the SERS signal intensity $(<10 \%$ in a relative standard deviation) is difficult to obtain, ${ }^{44}$ and the result could still vary for different analytes, solvents or assays. Another major bottleneck for real-time monitoring with SERS is the limitations related to reversibility and reusability of the sensors. Most of the SERS sensors are single use, which in addition increase the operational cost of a SERS-based assay. There are examples for recycling of SERS substrates, based on the replacement of metal structured components, ${ }^{45}$ washing procedures utilizing harsh reagents, thermal treatment where molecules are desorbed at high temperature, ${ }^{46} \mathrm{UV}$ irradiation ${ }^{47}$ or by application of high voltages; 48 these processes rely on drastic surface treatments, specialized equipment and time consuming reversion processes. To the best of our knowledge, all SERS substrate reusability methods aimed at quantitative SERS detection reported in literature solely rely on surface cleaning processes and/or metal re-deposition. Therefore, these methods are not applicable for real time, in-flow SERS measurements that require surface rejuvenation for multi-use, quantitative detection of trace amounts of analytes. Recently, an electrically assisted rejuvenation of SERS-active nanostructured silver surface by applying high voltages $(100 \mathrm{~V})^{48}$ for inline SERS detection have been shown, which indicates that regeneration of SERS substrates for quantitative molecular detection could potentially be achieved.

In order to facilitate the reusability of the SERS substrate within an assay in real time, we present the application of a highly uniform Au-capped Si nanopillar SERS substrate 32,49 in combination with electrochemistry. We demonstrate a single chip-based, electrochemical-SERS platform with significantly improved sensitivity capable to perform i) SERS assay calibration, ii) sensing and iii) SERS chip reusability in real-time. As a case study we focused on melamine a toxic compound used for adulteration of dairy products. Melamine is commonly measured using chromatographic techniques or immunoassays, ${ }^{50}$ but proved be suitable for SERS-based detection. ${ }^{42,51-57}$ When combined with sample pre-treatment, nanoparticle-based SERS substrates, were shown to reach a 
limit of detection of $0.03 \mathrm{ppm}$ in milk. ${ }^{58}$ However, nanoparticle-based SERS substrates often suffer from low substrate homogeneity and signal reproducibility, which can be overcome through purposefully engineered nanostructured SERS substrates. ${ }^{9,59}$ As an example P. Rajapandiyan et al. ${ }^{60}$ reported a detection limit for melamine in diluted milk of 2 ppm for SERS-substrates based on silvernanoparticle decorated nanorod structures. 


\section{Materials and Methods}

\section{Chemicals}

The supporting electrolyte for all samples was $0.1 \mathrm{M}$ phosphate buffer saline (PBS) containing, $0.01 \mathrm{M}$ disodium phosphate $\left(\mathrm{Na}_{2} \mathrm{HPO}_{4}\right), 0.001 \mathrm{M}$ monopotassium phosphate $\left(\mathrm{KH}_{2} \mathrm{PO}_{4}\right), 0.002 \mathrm{M}$ potassium chloride $(\mathrm{KCl})$ and $0.1 \mathrm{M}$ sodium chloride $(\mathrm{NaCl})$. The $\mathrm{pH}$ of the PBS $(\mathrm{pH} 7.4)$ was adjusted, when required, to $\mathrm{pH} 3.6$ with $10 \%$ hydrochloric acid $(\mathrm{HCl})$ using a standard pH-glass electrode (WTW series, Xylem Analytics, Xylem Inc., Rye Brook, NY, USA). Melamine stock solutions of $10 \mathrm{ppm}$ and $100 \mathrm{ppm}$ were prepared using melamine powder in PBS for both $\mathrm{pH} 3.6$ and pH 7.4. All working solutions were prepared with ultrapure water obtained from a Milli-Q purification system (Millipore Corporation, Billerica, MA, USA), and all the chemicals were purchased from Merck KGaA (Darmstadt, Germany).

\section{Milk samples and pre-treatment}

The milk samples with $0.5 \%$ fat content (Dansk Maelk, Jacobs Douwe Egberts Professional, København, Denmark) were purchased from a local commercial supplier. The samples, spiked with melamine, were pretreated prior to SERS measurement, using a gel filtration column (NAP-25, GE Healthcare, Pittsburgh, PA, USA) as also described by A. Kim et al. ${ }^{61}$ In short, $2.5 \mathrm{ml}$ milk samples, spiked with various melamine concentrations $(0.3 \mathrm{ppm}-2.5 \mathrm{ppm})$ using the $\mathrm{pH} 7.4$ stock solutions, were loaded into the saturated filtration column. The milk was allowed to sink into the column and subsequently eluted in $2.5 \mathrm{ml}$ fractions with PBS $\mathrm{pH}$ 7.4. For SERS measurements the $\mathrm{pH}$ of the fractions were adjusted with $10 \% \mathrm{HCl}$ to a $\mathrm{pH}$ value between 3 to 4 , which was verified with $\mathrm{pH}$ paper (Merck KGaA, Darmstadt, Germany). 


\section{Fabrication of Au-capped Si nanopillar SERS substrates}

Au-capped Si nanopillar SERS substrates were fabricated as previously described. ${ }^{49,62}$ In short, nanopillars were produced on a 4-inch Si wafer using reactive ion etching (Advanced Si Etcher, STS MESC Multiplex ICP). In a subsequent step Au caps, with a nominal thickness of $160 \mathrm{~nm}$, were formed on the pillars by e-beam evaporation (Wordentec QCL 800, Wordentec, Devon, UK) of Au at a rate of $10 \AA / \mathrm{s}$. The wafer was diced into chips of 6 × $8 \mathrm{~mm}$ using a Laser Micromachining tool (3D-Micromac AG, D-09126 Chemnitz, Germany).

\section{Electrochemical-SERS detection unit}

The manufactured electrochemical-SERS platform (supplementary information, Fig. S1) consists of four main components, namely (i) a microscope slide holder base, (ii) a SERS chip used as working electrode (WE) connected via a copper tape, (iii) the sample chamber (V = $325 \mu 1$ ) and (iv) an electrode adapter, with a platinum (GoodFellow, Huntingdon, UK) counter electrode (CE) (Ø $1 \mathrm{~mm})$, and silver/ silverchloride $(\mathrm{Ag} / \mathrm{AgCl})$ reference electrode $(\mathrm{RE})(\varnothing 0.5 \mathrm{~mm})$.

Parts i - iii were designed to be disposable, while the electrode adapter (iv) can be removed and reused. The holder base consists of a flat glass microscope slide (width $=25 \mathrm{~mm}$, length $=75 \mathrm{~mm}$, height $=1 \mathrm{~mm}$ ) covered by a $0.5 \mathrm{~mm}$ Poly(methyl methacrylate) (PMMA, Axxicon Moulds, Eindhoven, Netherlands) sheet, which can be tightly secured at the Raman systems microscope stage. A cavity in the PMMA sheet defines the placement area of the $6 \times 8 \mathrm{~mm}$ SERS substrate. The SERS substrate is fixed in the PMMA sheet as depicted in Fig. S1 C and partially covered with a strip of $1 \mathrm{~cm}$ wide adhesive copper tape (Farnell Danmark AS, Herlev, Denmark), enabling the connection of the SERS substrate to the potentiostat.

The sample chamber consists of two components; a 0.5 PMMA base ( $25 \times 25 \mathrm{~mm})$, with a central opening ( 4 x $4 \mathrm{~mm}$ ) for covering the SERS chip and defining the measurement area, and a $3 \mathrm{~mm}$ high 
PMMA chamber $(25 \times 25 \mathrm{~mm})$ with a central cavity $(10 \times 10 \mathrm{~mm})$ and side opening accommodating the removable electrode adapter. All PMMA components were manufactured by laser ablation (Epilog Mini $1830 \mathrm{~W}$, Epilog, USA) and connected to the underlying structures using a $0.15 \mathrm{~mm}$ thick pressure sensitive double-sided adhesive (PSA, ARcare 90106, Adhesive Research, Limerick, Ireland) cut with a Silhouette Cameo Plotter (Silhouette America, Inc., Utah, US).

The electrode adapter (iv), is an irregular cross shaped $5 \mathrm{~mm}$ thick unit manufactured from polydimethylsiloxane (PDMS). PDMS (DOWSIL ${ }^{\mathrm{TM}}$, Dow Silicons, Wiesbaden, Germany) was prepared from base polymer and curing agent at a standard 1:10 mixing ratio directly into a custommade mold manufactured from a $5 \mathrm{~mm}$ thick PMMA sheet by laser ablation. After removal of trapped air bubbles, PDMS was cured for 12 hours at $70{ }^{\circ} \mathrm{C}$ in an oven. The soft elastomeric material allows easy manipulation of the piece in lateral directions and assists in sealing the sample chamber, as the electrode adapter is placed into a fitting opening. Two electrodes (CE and RE) were placed through the PDMS piece in lateral direction and fixed in the center of the sample chamber. In all cases the potential was applied vs. the $\mathrm{Ag} / \mathrm{AgCl} \mathrm{RE}$.

\section{SERS measurements}

SERS measurements were carried out using a Raman microscope (Thermo Scientific DXRxi, Waltham, MA, USA) equipped with an electron multiplying charge coupled device and a $780 \mathrm{~nm}$ laser. Measurements were performed in liquid on non-leaned Au-capped nanopillars. It has been previously shown that SERS molecular detection can be performed using non-leaned NP substrates1, which was also theoretically investigated by K. Wu et al. ${ }^{49}$ Measurements were recorded with $5 \mathrm{~mW}$ laser power, exposure time of $0.05 \mathrm{~s}$, a $10 \mathrm{X}$ objective, a $50 \mu \mathrm{m}$ slit and a step size of $50 \mu \mathrm{m}$. SERS maps measuring $2 \mathrm{~mm} \times 3.8 \mathrm{~mm}$ (height $\mathrm{x}$ width) were measured on the sensor surface through the liquid sample repetitively. The nanostructures were characterized before and after measurements using a scanning electron microscope (Zeiss Supra VP 40 SEM, Jena, Germany). 
In the electrochemical-SERS platform, the potential was applied using a commercial potentiostat (CHI 1030a, CH Instruments Electrochemical Analyzer, CH Instruments Inc., Austin, TX, USA).

\section{Measurement procedure}

Prior to each measurement, the SERS chip was pre-conditioned to ensure wetting of the chip, since the SERS substrates are hydrophobic after fabrication. The pre-conditioning step (1 min, $-0.8 \mathrm{~V}$ and $1 \mathrm{~min}, 0.8 \mathrm{~V}$ applied potential) in PBS at the relevant $\mathrm{pH}$ was repeated three times to ensure a homogenous surface wetting as well and signal stability. At the optimal applied potential (-0.8 V), the measurements of standards and samples were carried out after the chosen potential was applied for 30 seconds prior to SERS mapping. Each SERS map took 3 minutes and 30 seconds to record. The surface charge was reversed by applying a positive potential $(0.8 \mathrm{~V})$ for 1 min immediately after the SERS measurements.

This process was repeated on average four times for each concentration measurement. For the evaluation of the reversibility efficiency, an additional SERS map was recorded at no applied potential.

\section{Data Analysis}

Data processing and quantitative analysis were performed with a custom-made toolbox extension in Matlab (2019a, MathWorks, Natick, USA). Baseline correction between $630-760 \mathrm{~cm}^{-1}$ was performed by a linear model that was estimated using the observations from $630-650 \mathrm{~cm}^{-1}$ and $740-$ $760 \mathrm{~cm}^{-1}$. The median value for peak intensity and mean-area under the curve, taking into account removal of outliers, for the top $20 \%$ of data was obtained for each SERS map. The calibration curve was constructed based on the measured mean-area under the curve at a defined peak. For data collection, 4 SERS maps were recorded for each sample and measurements were carried out in two 
separate chips $(n=8)$. For the points in the calibration curve in the case of curve at no applied potential, SERS maps were recorded for each individual chip once and four chips were used for each concentration $(n=4)$. 


\section{Results and Discussion}

Melamine has been used as adulterant in food for increasing the apparent protein content in milk. ${ }^{50}$ This toxic compound can cause severe kidney damage ${ }^{63}$ therefore, the United Nations food standard has set a threshold of $1 \mathrm{ppm}$ of melamine in powdered infant formula and $2.5 \mathrm{ppm}$ for other foodstuffs and animal feed.$^{64}$ Due to the importance of melamine in food safety and the fact that this compound has been thoroughly studied with Raman spectroscopic techniques, ${ }^{65}$ including a variety of SERS platforms, ${ }^{13,61}$ it was chosen as model analyte for our electrochemical-SERS detection platform. Melamine is neutral at physiological $\mathrm{pH}$ and does not have interaction with an uncharged SERS chip (Fig. 1A). However, with a $\mathrm{pKa}$ value of 5 and $\mathrm{pKb}$ value of 9 , this molecule can be protonated (positive net charge) in solutions of $\mathrm{pH}<5$ and deprotonated (negative net charge) in solutions of $\mathrm{pH}>9$. In the electrochemical-assisted SERS approach, the Au-capped nanopillar substrates were used as WE to enable precise control of the applied potential on the SERS substrate. By modulating the charges of the target molecule, we can control the interaction of melamine with the charged SERS substrate (Fig. 1A).

The electrochemical-SERS platform is presented in Fig. 1B, where the potential is applied on the WE (SERS chip) vs. the $\mathrm{Ag} / \mathrm{AgCl} \mathrm{RE}$. The $\mathrm{CE}$ and $\mathrm{RE}$ are stabilized in the center of the sample chamber through a reusable electrode adapter. The assembled detection chamber (Fig. 1C), can be easily handled and placed inside the Raman microscope (Fig. 1B). 

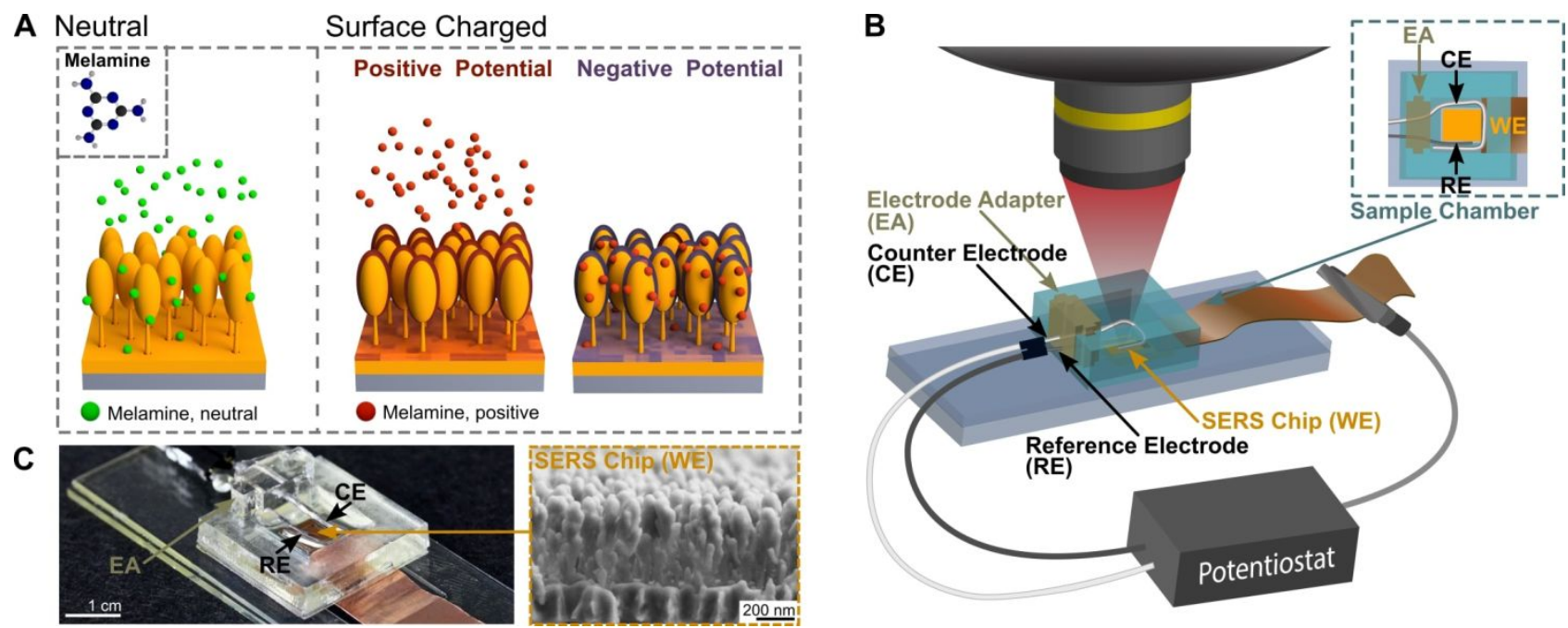

Figure 1: Working principle of the electrochemially assisted SERS-based detection showing the variations of surface charges on the Au-capped nanopillar SERS substrates and the suggested interaction of the surface with melamine (A). Illustration of the custom-made electrochemical-SERS platform and its respective system interfacing (B). Photo of the assembled detection chamber and SEM image of Au-capped nanopillar structures for SERS detection (C).

\section{Electrochemically enhanced SERS detection}

The melamine Raman spectrum was interpreted using Density Functional Theory (DFT) simulations and more detailed vibrational mode assignments are depicted in the supporting information Fig. S2 A. The Raman scattering signal from melamine powder (supplementary information, Fig. S2 B) shows distinct sharp peaks corresponding to the simulated results at $581 \mathrm{~cm}^{-}$ ${ }^{1}, 683 \mathrm{~cm}^{-1}$ and $985 \mathrm{~cm}^{-1}$. Nevertheless, when measuring melamine (2.5 ppm) spiked in milk (red line) at neutral (pH 7.4, Fig. 2A) and acidified (pH 3.6, Fig. 2B) pH on an Au-capped nanopillar SERS substrate no distinct melamine peak could be detected, due to surface fouling by the sample matrix. On the other hand, a measurable signal could be obtained for melamine at the same concentration in 0.1 M PBS (black line). 
A $\mathrm{pH}$ 7.4, Melamine neutral

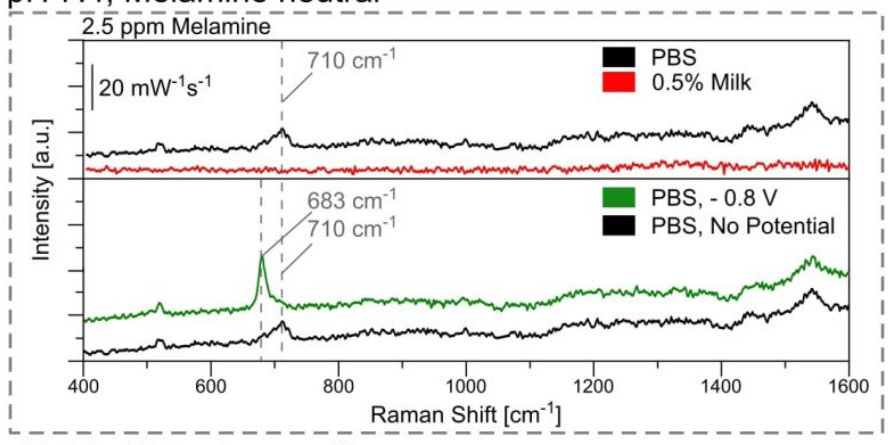

B $\mathrm{pH}$ 3.6, Melamine positive

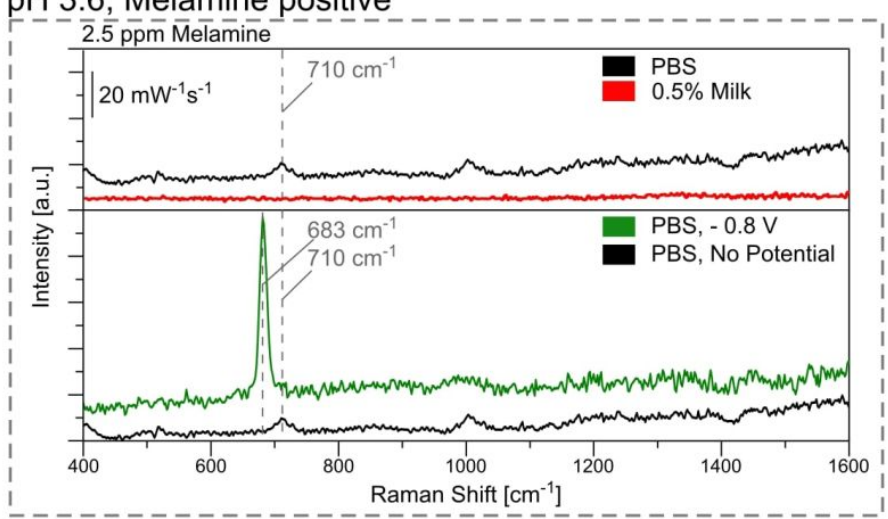

C 1 ppm Melamine positive, $\mathrm{pH} 3.6$

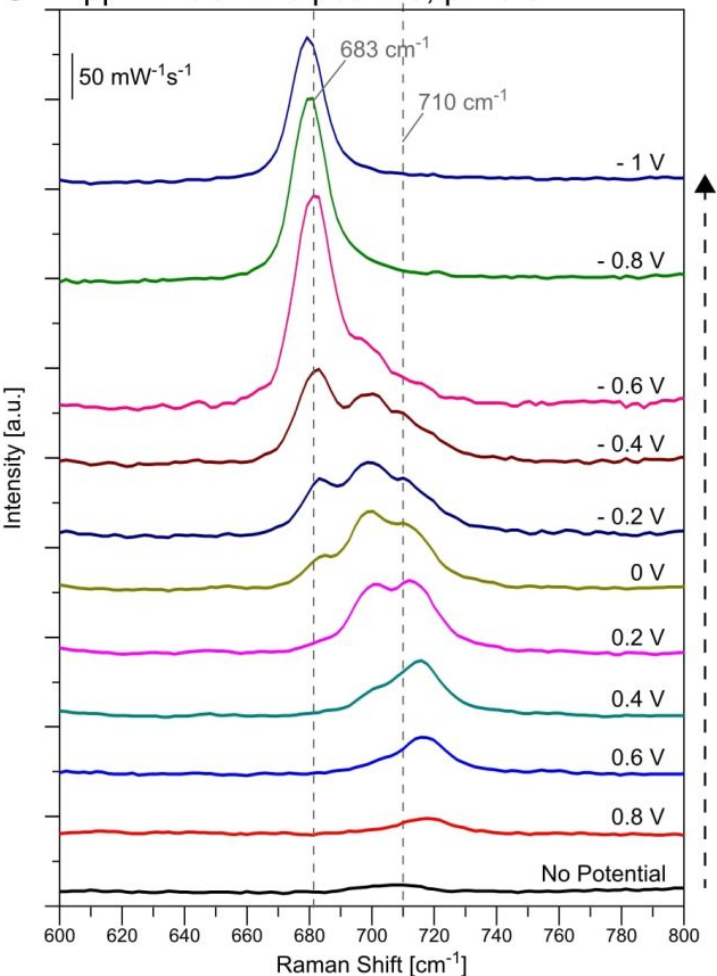

Figure 2: SERS spectra of $2.5 \mathrm{ppm}$ melamine in $\mathrm{PBS}$ and milk at a $\mathrm{pH} 7.4$ (A) and $\mathrm{pH} 3.6$ (B). Comparison of neutral and positively charged melamine in PBS at no (black line) and - $0.8 \mathrm{~V}$ vs. $\mathrm{Ag} / \mathrm{AgCl}$ (green line) applied potential. SERS spectra of $1 \mathrm{ppm}$ melamine in PBS at $\mathrm{pH} 3.6$ dependent on the applied potential (C). The SERS spectra have been shifted for clear representation.

The detected melamine peak at $\mathrm{pH} 7.4$ and $\mathrm{pH} 3.6$ in PBS was at a peak position around $710 \mathrm{~cm}^{-1}$, which was different from the signal recorded from the melamine powder (Fig. S2 A). This is probably due to a different state of melamine in the liquid environment. In Figure 2A, Raman spectra of $2.5 \mathrm{ppm}$ melamine dissolved in PBS ( $\mathrm{pH} 7.4$ ) at no applied potential (black line) and at high negative potential (- $0.8 \mathrm{~V}$, green line) are shown. At no applied potential, we recorded a peak attributed to melamine around $710 \mathrm{~cm}^{-1}$, while at $-0.8 \mathrm{~V}$ applied potential a peak at $683 \mathrm{~cm}^{-1}$ was measured. When measurements were performed in milk, no distinct peaks could be observed independent from $\mathrm{pH}$ and applied surface charge (supplementary information, Fig. S3) due to the turbidity of the sample matrix. 
Potential dependent Raman peak shifts have been previously reported by E. Koglin et al. ${ }^{66}$ and can be attributed to the interaction mechanism of melamine at the metal-electrolyte interface. In our case, the applied negative potential resulted in more pronounced peak shape, a peak shift and increased peak intensity for the same melamine concentration at $\mathrm{pH}$ 7.4. At $\mathrm{pH} 3.6$, the melamine molecule will have a controlled positive net charge. At this $\mathrm{pH}$ similar peak position shifts can be observed, as depicted in Fig. 2B (green line vs. black line). The observed peak intensity at $683 \mathrm{~cm}^{-1}$ is ten times higher at - $0.8 \mathrm{~V}$ applied potential (green line, Fig. 2A vs. Fig. 2B), in the case of the acidified solution in comparison to the solution at $\mathrm{pH}$ 7.4. The increase in intensity is caused by electrostatic attraction between the negatively charged SERS substrate at $-0.8 \mathrm{~V}$ and the protonated melamine molecules at $\mathrm{pH} 3.6{ }^{42}$ Despite of the signal increase at $\mathrm{pH} 3.6$ with - $0.8 \mathrm{~V}$ applied potential, when melamine was measured directly in milk samples there was no peak observed due to the turbidity of the solution caused by the lipid micellar components in the milk (Fig. 2B, black line vs. red line). This observation indicates a strong need for a simple sample pre-treatment when dealing with real samples.

As we demonstrated, the SERS-based detection of melamine is significantly enhanced due to electrostatic interaction between the negatively charged sensor surface at $-0.8 \mathrm{~V}$ applied potential and the positively charged analyte $(\mathrm{pH} 3.6)$. We observed that changes in surface charge cause a significant peak-pattern-change when measuring 1 ppm melamine in acidified PBS (pH 3.6, Fig. 2C). Examples of recorded maps for this measurements has been included in the (supplementary information, Fig. S4). Generally, the peak intensities increase with increased negative applied potential from $-0.2 \mathrm{~V}$ up to $-0.8 \mathrm{~V}$, followed by a slight decrease in intensity at $-1 \mathrm{~V}$. Similar effects have been shown by A.M. Robinson et al. ${ }^{42} \mathrm{We}$ also observed that up to $0.4 \mathrm{~V}$ applied potential the peak at $710 \mathrm{~cm}^{-1}$ is dominant, which has also been previously reported when measuring melamine in aqueous solutions. ${ }^{42,61}$ However, at $0.2 \mathrm{~V}$ an additional second peak appears around $702 \mathrm{~cm}^{-1}$, while at $0 \mathrm{~V}$ the peak at $683 \mathrm{~cm}^{-1}$ becomes apparent and intensifies with increased negative applied 
potential, while the $702 \mathrm{~cm}^{-1}$ and $710 \mathrm{~cm}^{-1}$ peaks start to disappear. At an applied potential of $-0.6 \mathrm{~V}$ the $683 \mathrm{~cm}^{-1}$ peak is the major spectral peak and it reaches its highest intensity at $-0.8 \mathrm{~V}$. Therefore, for further experiment aimed for melamine quantification, - $0.8 \mathrm{~V}$ applied potential was used.

\section{Reversibility of the SERS substrate}

T.-A. Meier et al. ${ }^{48}$ has previously reported a fast electrically assisted regeneration of a stationary SERS substrate integrated into a microfluidic channel via short-term application of $100 \mathrm{~V}$ to the surface. The regenerated crystal violet $\left(10^{-5} \mathrm{M}\right)$ SERS spectra displayed a $\sim 33 \%$ deviation in spectral intensity possibly caused by morphology changes of the Ag substrate induced through the high applied voltage during the desorption process, which is a major limitation for utilizing the method in quantitative SERS applications reliably. In this work, we evaluated the possibility of reversing the electrical attraction on the surface, by applying a positive potential of $0.8 \mathrm{~V}$ for 1 min after measurements, aiming to break the interaction between the analyte and the SERS substrate (Fig. 3A).

In Fig. 3B, SERS spectra of 1 ppm melamine at multiple repetition cycles in acidified PBS (pH 3.6) are shown. The spectra were recorded on a single chip while the applied potential was switched between - $0.8 \mathrm{~V}$ and $0.8 \mathrm{~V}$ (Fig. 3C). After each measurement at - $0.8 \mathrm{~V}$ applied potential, the analyte was electrostatically removed from the surface of the SERS substrate when $0.8 \mathrm{~V}$ was applied for 1 min. Raman signal intensities of the $683 \mathrm{~cm}^{-1}$ peak are stable throughout the measurements at $0.8 \mathrm{~V}$ applied potential (Fig. 3B, red curves). The black curves (Fig. 3B) are the intermediate measurements performed at no applied potential, after the reversal $(0.8 \mathrm{~V}, 1 \mathrm{~min})$ step, and show that melamine was efficiently removed from the SERS substrate. There is a clear and stable $710 \mathrm{~cm}^{-1}$ peak at no applied potential, while at a highly negative applied potential a dominant peak at $683 \mathrm{~cm}^{-1}$ could be observed. Both peaks are characteristic for the applied potential state (Fig. 2C), which shows that the substrate returned to its initial state after charge reversal. Throughout the repetitions cycles no 
additional spectral peaks could be observed (supplementary information, Fig. S5 A). The effect of applied potentials on the morphology of SERS substrates was negligible, see SEM images of the SERS substrates before and after an experimental run depicted in Fig. S5 B (supplementary information).

In Fig. 3C the variation between measurements is shown, considering the area under the curve, for the $683 \mathrm{~cm}^{-1}$ peak, at no applied potential (black) and at $-0.8 \mathrm{~V}$ applied potential (red). To ensure accurate detection achievable on a uniformly charged and wetted surface, a pre-conditioning step (two repetition cycles of - 0.8 and $0.8 \mathrm{~V}$ applied potential) was introduced prior an assay, as indicated in the 'Measurement procedure' section and therefore the data from the pre-conditioning step was not included in the signal reversibility study. When considering the peak intensities at $-0.8 \mathrm{~V}$ applied potential for the $683 \mathrm{~cm}^{-1}$ peak we obtained a $23.7 \%$ relative standard variation between the samples. However, when calculating the area under the curve for the $683 \mathrm{~cm}^{-1}$, as shown in Fig. $3 \mathrm{C}$, the variation, calculated as relative standard deviation (RSD), was lower (11.4\%) probably because in this case the software included in the calculation also the contribution of the $710 \mathrm{~cm}^{-1}$ peak area. 


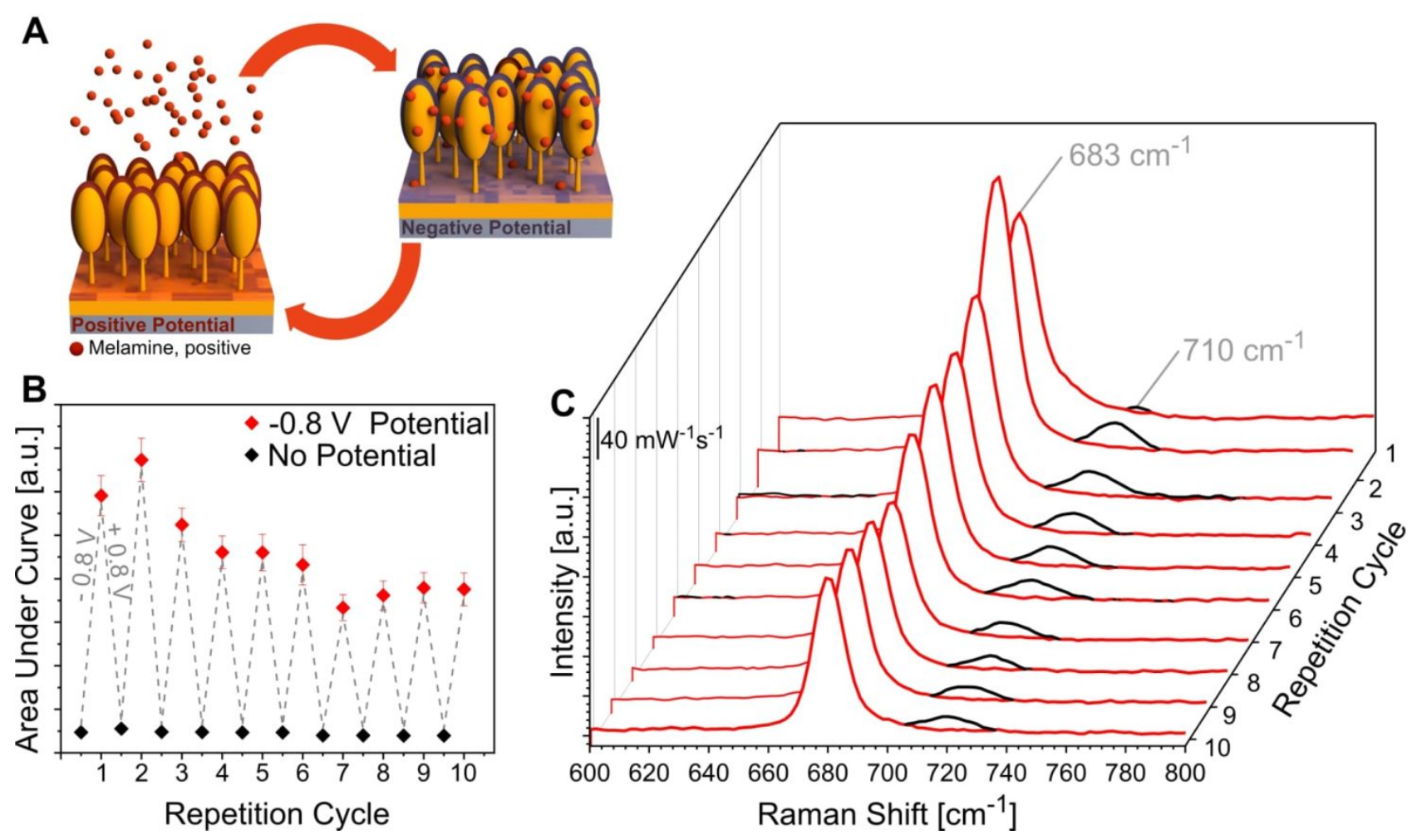

Figure 3: Working principle of the surface reversibility (A). Reversibility study of 1 ppm melamine in acidified PBS, data recorded at $-0.8 \mathrm{~V}$ applied potential vs $\mathrm{Ag} / \mathrm{AgCl}$ and at no applied potential (B); inset shows the area under curve at the $683 \mathrm{~cm}^{-1}$ peak $(C) .(\mathrm{n}=4)$

\section{Calibration on a single chip}

Due to the electrochemically controlled reversibility of the substrate, it was possible to perform a full calibration curve on a single SERS substrate (Fig. 4, blue dots). Each concentration was measured repeatedly on the SERS substrate, followed by a complete exchange of the sample between concentrations after final analyte repulsion.

For comparison, we constructed a calibration curve at no applied potential with an uncharged analyte (Fig. 4, black dots), which required 60 separate Au-capped nanopillar chips to obtain comparable numbers of data point as with the electrochemically assisted approach. 


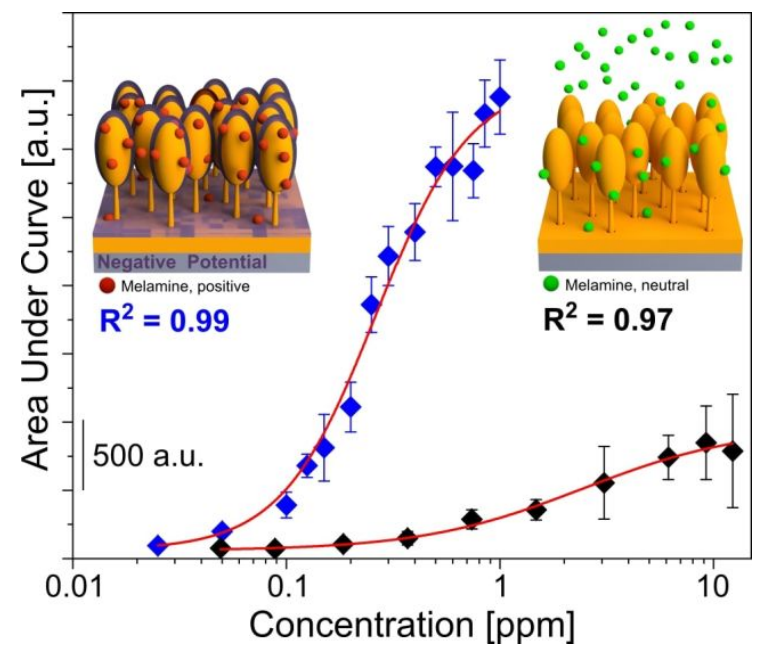

Figure 4: Calibration curve of melamine in PBS performed using the electrochemically assisted SERS-platform (blue, $\mathrm{pH} 3.6, \mathrm{n}=8$ ) compared to calibration curve obtained with individual chips for each concentration (black, $\mathrm{pH} 7.4, \mathrm{n}=4$ ).

There is a clear difference between the calibration curves presented in Fig. 4, with an improved sensitivity in the case of the electrochemically enhanced SERS. The linear range in this case was between 0.05 and $0.5 \mathrm{ppm}$ with the detection limit of $0.01 \mathrm{ppm}$. Based on the calibration curve it can be seen that with non-charged melamine and at no applied potential, the detection limit is $0.5 \mathrm{ppm}$, which is already 20 times lower than previously reported detection limits for melamine of $10 \mathrm{ppm}$ on our Au-capped nanopillar substrates ${ }^{13}$ and 10 times lower than on other spectroelectrochemical SERS systems utilizing screen printed electrodes modified with silver nanoparticles. ${ }^{42}$ As shown in Fig. 4 , we obtained calibration curves on a single SERS chip by utilizing $60-80$ repetition cycles on various melamine concentrations. Additionally, the RSD when using the electrochemically assisted approach was lower $14.1 \%$ then when working with individual chips for the calibration curve (30\% RSD). We evaluated the efficiency of signal reversibility, since it is a crucial factor during sensor calibration and when performing an assay. If melamine is not removed efficiently, it can occur that the molecules will gradually accumulate on the sensor substrate leading to an artificial signal increase and a false positive result. 
To verify that melamine was removed, through electrostatic $(0.8 \mathrm{mV})$ repulsion, after detection, we measured acidified PBS control (0 ppm melamine) several times at $-0.8 \mathrm{mV}$, namely after preconditioning, intermediately between concentrations and at the end of a measurement set and after each single-chip calibration. The SERS spectra of acidified PBS controls for three separate calibration experiments are shown in Fig. 5. We observed no significant melamine-related Raman peaks $\left(683 \mathrm{~cm}^{-1}\right.$ or $\left.710 \mathrm{~cm}^{-1}\right)$ in acidified PBS controls for any of the experiments. This strongly suggest that the electrostatic repulsion of protonated melamine from the SERS substrate when applying a highly positive surface potential $(0.8 \mathrm{~V})$ functions reliable and additionally allows efficient sample removal from the sensor surface. During sample exchange, melamine could be removed from the sample chamber without any residues avoiding cross contamination between samples and artificially increased signal intensities.

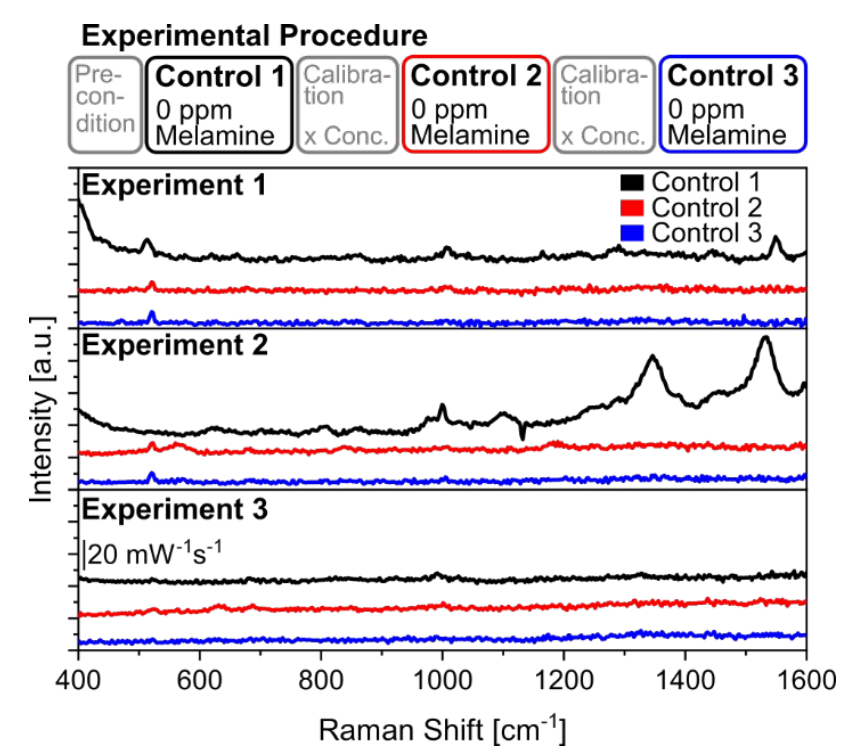

Figure 5: SERS spectra of acidified PBS for three separate calibration curve experiments. The data was recorded at $-0.8 \mathrm{~V}$ applied potential vs. $\mathrm{Ag} / \mathrm{AgCl}$ after sample pre-conditioning, in the middle and at the end of a calibration curve experimental run $(n=6)$. The SERS spectra have been shifted for clear representation. 


\section{Melamine detection in milk}

Direct application of sensors in real samples, such as milk, can be challenging, considering the effect of interfering compounds, which either can foul the sensor surface or lead to false positive outputs. ${ }^{13,38,41,42,61,67}$ For the detection of melamine, liquid chromatography or gas chromatography coupled with mass spectrometry as well as immunosorbent assays are commonly used..$^{50}$ However, these techniques often require complex sample pre-treatment procedures, such as chromatographic separation and phase extraction, ${ }^{68}$ procedures as well as well-trained personal and costly analysis systems. SERS on the other hand, proved to be a feasible alternative for fast and cost efficient melamine detection ${ }^{42,51-57}$ and has been successfully used in combination with various sample pretreatment approaches, ${ }^{11,57,69}$ which proved to be important when quantifying analytes from a complex sample matrix.

Milk is a commonly used staple food with a rather complex matrix, it is composed of next to $90 \%$ water, different proportions of lipids, proteins, carbohydrates as well as small amounts of minerals and other micronutrients. ${ }^{70}$ Common simple pre-treatment approaches for melamine separation from milk utilized in combination with SERS-detection include centrifugation ${ }^{71,72}$ and dilution. Melamine was measured from milk using a variety of nanoparticle-based SERS substrates,${ }^{50}$ reaching detection limits down to $0.03 \mathrm{ppm}^{58}$ when used in combination with pre-treatment technologies.

In this work we utilized a simple sample pre-treatment based on solid phase separation of milk on a gel filtration column, previously introduced by A. Kim et al. ${ }^{61}$ We optimized the fraction collection step, using milk spiked with 10 ppm melamine.

The first two fractions were discarded and not used for SERS based detection, since they contained the majority of milk micelles, resulting in a high sample turbidity and lack of melamine signal (Fig. 6A). In Fig. 6B it is shown that we obtain a clear melamine signal from fraction 3, while there 
is no detectable melamine form milk spiked with melamine. As can be seen in Fig. 6C, the majority of melamine is eluted in fraction 3 and 4, which were combined for further analysis. Using the calibration curve shown in Fig. 4 (pH 7.4, no applied potential) we found that the concentration in the combined fractions 3 and 4 was $9.3 \pm 0.8 \mathrm{ppm}$. Melamine detected from the milk fractions shows a slightly lower response than the signal recorded from spiked PBS solutions, indicating that a part of the analyte was either retained in the gel filtration column or eluted in other fractions.
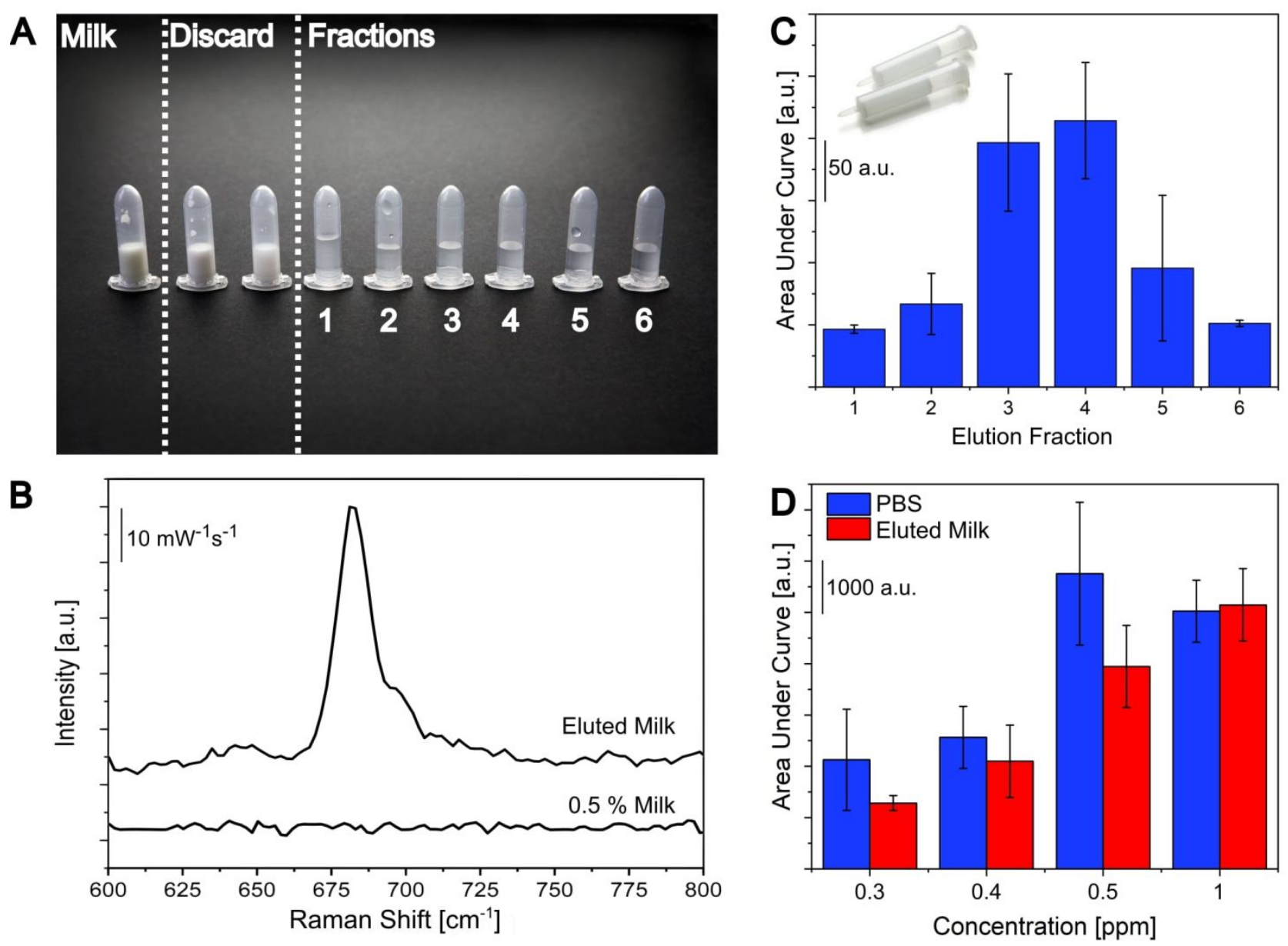

Figure 6: Photo of milk in comparison with the obtained eluted fractions (A). SERS spectra of milk and signal recorded from the combined elution fraction 3 and 4 under no applied potential conditions (B). The SERS spectra have been shifted for clear representation. Area under the curve for $710 \mathrm{~cm}^{-1}$ melamine peak at no applied potential conditions for fractions 1 to $6(\mathrm{C}, \mathrm{n}=3)$. Signal recorded for 
melamine detected from milk and PBS after elution using electrochemically assisted SERS (D). Peak areas for $683 \mathrm{~cm}^{-1}$ melamine peak at $-0.8 \mathrm{~V}$ applied potential are shown. $(\mathrm{n}=8)$.

Once we established the extraction of melamine from milk, we evaluated the efficiency of the sample pre-treatment method combined with the electrochemically assisted SERS-based detection by measuring various concentrations (0.3 to $1 \mathrm{ppm})$ of melamine spiked in milk and PBS (Fig. 6D). After elution, the combined fractions $(3 \& 4)$ were acidified with $10 \% \mathrm{HCl}$ and measurements were performed at - $0.8 \mathrm{~V}$ applied potential with intermediate reversal of charges. Melamine detected from the milk fractions shows a slightly lower response than the signal recorded from spiked PBS solutions, indicating that a part of the analyte probably was retained in the gel filtration column. We found that approximately $90 \%$ of the melamine was eluted for all tested concentrations, leading to a reliable detection of at least $0.3 \mathrm{ppm}$ melamine from milk samples, which is below the lowest set limit of 1 ppm. Kim et al. ${ }^{61}$ earlier reported a detection limit of $1 \mathrm{ppm}$ melamine in milk with the same gel filtration sample pre-treatment method using SERS substrates. In addition, electrochemically assisted SERS-based detection of melamine in milk has been demonstrated by C.L. Brosseau et al. with a portable system with $5 \mathrm{ppm}$ melamine detection limit in milk. ${ }^{71}$ An added advantage of the here presented method beside the lower detection limits is the reversibility of the SERS-based detection, which substantially lowers the number of sensors needed in a quantitative assay. 


\section{Conclusion}

In this paper, we present a cost efficient and specific approach for melamine detection, using a compact and easy to use detection platform. The technique allows reliable, repetitive measurements on a single SERS chip, which eliminated chip-to-chip variations and greatly decrease the cost of analysis. The obtained limit of detection $(0.01 \mathrm{ppm})$ for melamine in PBS, and the lowest measured concentration in milk $(0.3 \mathrm{ppm})$ are below the maximum allowed levels in powdered infant formula of $1 \mathrm{ppm}{ }^{64}$ This indicates that when combined with the suitable sample pre-treatment step the here presented electrochemically assisted SERS assay could be suitable for screening of infant formula. Our application of a simple sample pre-treatment enables melamine detection from real samples without the need of sample dilution. The presented method and detection platform open up new possibilities for applications requiring continuous monitoring and in-line detection, considering the reversible nature of detection. Additionally the presented system would be suitable for on-site detection when combined with miniaturized Raman systems ${ }^{19,21}$ and potentiostats. ${ }^{73,74}$ The introduced pre-treatment and reversible detection unit can be easily integrated as automated units for continuous detection in an industrial set-up.

\section{Acknowledgement}

We wish to thank Jesper Scheel for beautiful photos and Ellen Vallentin Christiansen for assistance in illustration preparations. This work was funded by the NAPLAS, HERMES and IDUN project, the Danish Council for Independent Research as well as the European Research Council under the European Union's Seventh Framework Programme (FP7/2007-2013) / ERC grant agreement 320535. 


\section{Supporting Information}

Additional material about components of the electrochemical-SERS platform, theoretical simulations of the ground molecular structure and vibrational spectrum of melamine, direct melamine detection from milk and signal reversibility.

\section{References}

(1) Chung, T.; Lee, S. Y.; Song, E. Y.; Chun, H.; Lee, B. Plasmonic Nanostructures for NanoScale Bio-Sensing. Sensors 2011, 11 (11), 10907-10929. https://doi.org/10.3390/s111110907.

(2) Giljohann, D. A.; Mirkin, C. A. Drivers of Biodiagnostic Development; Nature Publishing Group, 2009; Vol. 462, pp 461-464. https://doi.org/10.1038/nature08605.

(3) Reiner, J. E.; Balijepalli, A.; Robertson, J. W. F.; Campbell, J.; Suehle, J.; Kasianowicz, J. J. Disease Detection and Management via Single Nanopore-Based Sensors. Chem. Rev. 2012, 112 (12), 6431-6451. https://doi.org/10.1021/cr300381m.

(4) Ziegler, L. D. Biosensing Applications of SERS: Bacterial Diagnostics, Blood Aging and Forensics. In Advanced Photonics 2015; OSA: Washington, D.C., 2015; p SeT1C.1. https://doi.org/10.1364/SENSORS.2015.SeT1C.1.

(5) Premasiri, W. R.; Chen, Y.; Fore, J.; Brodeur, A.; Ziegler, L. D. SERS Biomedical Applications: Diagnostics, Forensics, and Metabolomics. In Frontiers and Advances in Molecular Spectroscopy; Elsevier, 2018; pp 327-367. https://doi.org/10.1016/B978-0-12811220-5.00010-1.

(6) Baia, M.; Astilean, S.; Iliescu, T. Raman and SERS Investigations of Pharmaceuticals; Springer Berlin Heidelberg: Berlin, Heidelberg, 2008. https://doi.org/10.1007/978-3-54078283-4. 
(7) Wei, H.; Hossein Abtahi, S. M.; Vikesland, P. J. Plasmonic Colorimetric and SERS Sensors for Environmental Analysis. Environ. Sci. Nano 2015, 2 (2), 120-135. https://doi.org/10.1039/C4EN00211C.

(8) Xie, X.; Pu, H.; Sun, D.-W. Recent Advances in Nanofabrication Techniques for SERS Substrates and Their Applications in Food Safety Analysis. Crit. Rev. Food Sci. Nutr. 2018, 58 (16), 2800-2813. https://doi.org/10.1080/10408398.2017.1341866.

(9) Mosier-Boss, P.; Mosier-Boss; A., P. Review of SERS Substrates for Chemical Sensing. Nanomaterials 2017, 7 (6), 142. https://doi.org/10.3390/nano7060142.

(10) Morelli, L.; Serioli, L.; Centorbi, F. A.; Jendresen, C. B.; Matteucci, M.; Ilchenko, O.; Demarchi, D.; Nielsen, A. T.; Zór, K.; Boisen, A. Injection Molded Lab-on-a-Disc Platform for Screening of Genetically Modified: E. Coli Using Liquid-Liquid Extraction and Surface Enhanced Raman Scattering. Lab Chip 2018, $18 \quad$ (6), 869-877. https://doi.org/10.1039/c7lc01217a.

(11) Morelli, L.; Andreasen, S. Z.; Jendresen, C. B.; Nielsen, A. T.; Emnéus, J.; Zór, K.; Boisen, A. Quantification of a Bacterial Secondary Metabolite by SERS Combined with SLM Extraction for Bioprocess Monitoring. Analyst 2017, $142 \quad$ (23), 4553-4559. https://doi.org/10.1039/c7an01393k.

(12) Martin, J. W.; Nieuwoudt, M. K.; Vargas, M. J. T.; Bodley, O. L. C.; Yohendiran, T. S.; Oosterbeek, R. N.; Williams, D. E.; Cather Simpson, M. Raman on a Disc: High-Quality Raman Spectroscopy in an Open Channel on a Centrifugal Microfluidic Disc. Analyst 2017, 142 (10), 1682-1688. https://doi.org/10.1039/C6AN00874G.

(13) Durucan, O.; Rindzevicius, T.; Schmidt, M. S.; Matteucci, M.; Boisen, A. Nanopillar Filters 
for Surface-Enhanced Raman Spectroscopy. ACS Sensors 2017, 2 (10), 1400-1404. https://doi.org/10.1021/acssensors.7b00499.

(14) Popp, J. Chip-Based Raman Spectroscopy for Biomedical Diagnosis. In Optics Infobase Conference Papers; 2015; Vol. 2015.

(15) SERS Substrates - Low cost and high performing SERS substrate, SERStrate, from Silmeco http://www.silmeco.com/products/sers-substrate-serstrate/ (accessed Jun 13, 2019).

(16) SERS Substrates - StellarNet, Inc. https://www.stellarnet.us/spectrometers-accessories/serssubstrates/ (accessed Jun 13, 2019).

(17) Surface Enhanced Raman Spectroscopy (SERS) for Food Analysis » SPECTROSCOPY.TV http://spectroscopytv.com/sers-for-food-analysis/ (accessed Jun 13, 2019).

(18) Young, M. A.; Stuart, D. A.; Lyandres, O.; Glucksberg, M. R.; Van Duyne, R. P. SurfaceEnhanced Raman Spectroscopy with a Laser Pointer Light Source and Miniature Spectrometer. Can. J. Chem. 2004, 82 (10), 1435-1441. https://doi.org/10.1139/v04-098.

(19) Park, M. H.; Jang, Y. J.; Sung-suh, H. M.; Sung, M. M. Selective Atomic Layer Deposition of Titanium Oxide on Patterned Self-Assembled Monolayers Formed by Microcontact Printing. 2004, No. 18, 2257-2260.

(20) Xuan Quang, L.; Lim, C.; Hun Seong, G.; Choo, J.; Jun Do, K.; Yoo, S.-K. A Portable SurfaceEnhanced Raman Scattering Sensor Integrated with a Lab-on-a-Chip for Field Analysis. https://doi.org/10.1039/b808835g.

(21) Perikin Elmer. New Portable Raman Spectrometer. Mater. Today 2008, 11 (10), 47. https://doi.org/10.1016/S1369-7021(08)70219-0. 
(22) Li, D.; Li, D.-W.; Fossey, J. S.; Long, Y.-T. Portable Surface-Enhanced Raman Scattering Sensor for Rapid Detection of Aniline and Phenol Derivatives by On-Site Electrostatic Preconcentration. Anal. Chem. 2010, 82 (22), 9299-9305. https://doi.org/10.1021/ac101812x.

(23) Foster, B. Portable Raman Microscopy-Laboratory-Quality Results Measured Anywhere. Am. Lab. 2015, 47 (3).

(24) Han, Z.; Liu, H.; Meng, J.; Yang, L.; Liu, J. J.; Liu, J. J. Portable Kit for Identification and Detection of Drugs in Human Urine Using Surface-Enhanced Raman Spectroscopy. Anal. Chem. 2015, 87 (18), 9500-9506. https://doi.org/10.1021/acs.analchem.5b02899.

(25) Chen, Y.; Chen, Z.-P.; Zuo, Q.; Shi, C.-X.; Yu, R.-Q. Surface-Enhanced Raman Spectroscopy Based on Conical Holed Enhancing Substrates. Anal. Chim. Acta 2015, 887, 45-50. https://doi.org/10.1016/J.ACA.2015.07.025.

(26) Betz, J. F.; Yu, W. W.; Cheng, Y.; White, I. M.; Rubloff, G. W. Simple SERS Substrates: Powerful, Portable, and Full of Potential. Phys. Chem. Chem. Phys. 2014, 16 (6), 2224-2239. https://doi.org/10.1039/c3cp53560f.

(27) Goodacre, R.; Graham, D.; Faulds, K. Recent Developments in Quantitative SERS: Moving towards Absolute Quantification. TrAC Trends Anal. Chem. 2018, 102, 359-368. https://doi.org/10.1016/J.TRAC.2018.03.005.

(28) Tian, L.; Su, M.; Yu, F.; Xu, Y.; Li, X.; Li, L.; Liu, H.; Tan, W. Liquid-State Quantitative SERS Analyzer on Self-Ordered Metal Liquid-like Plasmonic Arrays. https://doi.org/10.1038/s41467-018-05920-z.

(29) Yu, B.; Ge, M.; Li, P.; Xie, Q.; Yang, L. Development of Surface-Enhanced Raman Spectroscopy Application for Determination of Illicit Drugs: Towards a Practical Sensor. 
Talanta 2019, 191, 1-10. https://doi.org/10.1016/j.talanta.2018.08.032.

(30) Sharma, B.; Frontiera, R. R.; Henry, A.-I.; Ringe, E.; Van Duyne, R. P. SERS: Materials, Applications, and the Future Background and Mechanism; 2012; Vol. 15. https://doi.org/10.1016/S1369-7021(12)70017-2.

(31) Brown, R. J. C.; Milton, M. J. T. Nanostructures and Nanostructured Substrates for SurfaceEnhanced Raman Scattering (SERS). J. Raman Spectrosc. 2008, 39 (10), 1313-1326. https://doi.org/10.1002/jrs.2030.

(32) Schmidt, M. S.; Hübner, J.; Boisen, A. Two-Step Fabrication of Metal-Coated Silicon Nanopillars with Large Raman Enhancement. AIP Conf. Proc. 2010, 1267, 912-913. https://doi.org/10.1063/1.3482886.

(33) Pazos-Pérez, N.; Ni, W.; Schweikart, A.; Alvarez-Puebla, R. A.; Fery, A.; Liz-Marzán, L. M. Highly Uniform SERS Substrates Formed by Wrinkle-Confined Drying of Gold Colloids. Chem. Sci. 2010, 1 (2), 174. https://doi.org/10.1039/c0sc00132e.

(34) Yuan, Y.; Panwar, N.; Yap, S. H. K.; Wu, Q.; Zeng, S.; Xu, J.; Tjin, S. C.; Song, J.; Qu, J.; Yong, K. T. SERS-Based Ultrasensitive Sensing Platform: An Insight into Design and Practical Applications. Coord. Chem. Rev. 2017, 337, 1-33. https://doi.org/10.1016/j.ccr.2017.02.006.

(35) Schatz, G. C. C.; Young, M. A. A.; Van Duyne, R. P. P.; Duyne, R. P. Electromagnetic Mechanism of SERS. Top. Appl. Phys. 2006, 103, 19-46. https://doi.org/10.1007/11663898_2.

(36) Abdelsalam, M. E.; Bartlett, P. N.; Baumberg, J. J.; Cintra, S.; Kelf, T. A.; Russell, A. E. Electrochemical SERS at a Structured Gold Surface. Electrochem. commun. 2005, 7 (7), 740744. https://doi.org/10.1016/j.elecom.2005.04.028. 
(37) Zhao, L.; Blackburn, J.; Brosseau, C. L. Quantitative Detection of Uric Acid by Electrochemical-Surface Enhanced Raman Spectroscopy Using a Multilayered Au/Ag Substrate. Anal. Chem. 2015, 87 (1), 441-447. https://doi.org/10.1021/ac503967s.

(38) Goodall, B. L.; Robinson, A. M.; Brosseau, C. L. Electrochemical-Surface Enhanced Raman Spectroscopy (E-SERS) of Uric Acid: A Potential Rapid Diagnostic Method for Early Preeclampsia Detection. Phys. Chem. Chem. Phys. 2013, 15 (5), 1382-1388. https://doi.org/10.1039/c2cp42596c.

(39) Greene, B. H. C.; Alhatab, D. S.; Pye, C. C.; Brosseau, C. L. Electrochemical-Surface Enhanced Raman Spectroscopic (EC-SERS) Study of 6-Thiouric Acid: A Metabolite of the Chemotherapy Drug Azathioprine. J. Phys. Chem. C 2017, 121 (14), 8084-8090. https://doi.org/10.1021/acs.jpcc.7b01179.

(40) Bindesri, S. D.; Alhatab, D. S.; Brosseau, C. L. Development of an Electrochemical SurfaceEnhanced Raman Spectroscopy (EC-SERS) Fabric-Based Plasmonic Sensor for Point-of-Care Diagnostics. Analyst 2018, 143 (17), 4128-4135. https://doi.org/10.1039/c8an01117f.

(41) Lynk, T. P.; Sit, C. S.; Brosseau, C. L. Electrochemical Surface-Enhanced Raman Spectroscopy as a Platform for Bacterial Detection and Identification. Anal. Chem. 2018, 90 (21), 12639-12646. https://doi.org/10.1021/acs.analchem.8b02806.

(42) Robinson, A. M.; Harroun, S. G.; Bergman, J.; Brosseau, C. L. Portable Electrochemical Surface-Enhanced Raman Spectroscopy System for Routine Spectroelectrochemical Analysis. Anal. Chem. 2012, 84 (3), 1760-1764. https://doi.org/10.1021/ac2030078.

(43) O`Boyle, N. M.; Tenderholt, A. L.; Langner, K. M. Cclib: A Library for Package-Independent Computational Chemistry Algorithms. J. Comput. Chem. 2008, 29 (5), 839-845. 
https://doi.org/10.1002/jcc.20823.

(44) Wu, K.; Rindzevicius, T.; Schmidt, M. S.; Thilsted, A. H.; Boisen, A. Optimizing SilverCapped Silicon Nanopillars to Simultaneously Realize Macroscopic, Practical-Level SERS Signal Reproducibility and High Enhancement at Low Costs. J. Raman Spectrosc. 2017, 48 (12), 1808-1818. https://doi.org/10.1002/jrs.5255.

(45) Wu, K.; Li, T.; Schmidt, M. S.; Rindzevicius, T.; Boisen, A.; Ndoni, S. Gold Nanoparticles Sliding on Recyclable Nanohoodoos-Engineered for Surface-Enhanced Raman Spectroscopy. Adv. Funct. Mater. 2018, $28 \quad$ (2), 1-11. https://doi.org/10.1002/adfm.201704818.

(46) Sinha, G.; Depero, L. E.; Alessandri, I. Recyclable SERS Substrates Based on Au-Coated ZnO Nanorods. ACS Appl. Mater. Interfaces 2011, 3 (7), 2557-2563. https://doi.org/10.1021/am200396n.

(47) Weng, X.; Feng, Z.; Guo, Y.; Feng, J.; Hudson, S. P.; Zheng, J.; Ruan, Y.; Laffir, F.; Pita, I. Recyclable SERS Substrates Based on Fe 2 O 3 - Ag Hybrid Hollow Microspheres with Crumpled. 2016, 5238-5244. https://doi.org/10.1039/c6nj00473c.

(48) Meier, T. A.; Poehler, E.; Kemper, F.; Pabst, O.; Jahnke, H. G.; Beckert, E.; Robitzki, A.; Belder, D. Fast Electrically Assisted Regeneration of On-Chip SERS Substrates. Lab Chip 2015, 15 (14), 2923-2927. https://doi.org/10.1039/c5lc00397k.

(49) Wu, K.; Rindzevicius, T.; Schmidt, M. S.; Mogensen, K. B.; Xiao, S.; Boisen, A. Plasmon Resonances of Ag Capped Si Nanopillars Fabricated Using Mask-Less Lithography. Opt. Express 2015, 23 (10), 12965. https://doi.org/10.1364/OE.23.012965.

(50) Ritota, M.; Manzi, P. Melamine Detection in Milk and Dairy Products: Traditional Analytical 
Methods and Recent Developments. Food Anal. Methods 2018, 11 (1), 128-147. https://doi.org/10.1007/s12161-017-0984-1.

(51) Kim, A.; Barcelo, S. J.; Williams, R. S.; Li, Z. Melamine Sensing in Milk Products by Using Surface Enhanced Raman Scattering. Anal. Chem. 2012, 84 (21), 9303-9309. https://doi.org/10.1021/ac302025q.

(52) Hu, Y.; Feng, S.; Gao, F.; Li-Chan, E. C. Y.; Grant, E.; Lu, X. Detection of Melamine in Milk Using Molecularly Imprinted Polymers-Surface Enhanced Raman Spectroscopy. Food Chem. 2015, 176, 123-129. https://doi.org/10.1016/J.FOODCHEM.2014.12.051.

(53) Roy, P. K.; Huang, Y.-F.; Chattopadhyay, S. Detection of Melamine on Fractals of Unmodified Gold Nanoparticles by Surface-Enhanced Raman Scattering. J. Biomed. Opt. 2013, 19 (1), 011002. https://doi.org/10.1117/1.JBO.19.1.011002.

(54) Giovannozzi, A. M.; Rolle, F.; Sega, M.; Abete, M. C.; Marchis, D.; Rossi, A. M. Rapid and Sensitive Detection of Melamine in Milk with Gold Nanoparticles by Surface Enhanced Raman Scattering. Food Chem. 2014, 159, 250-256.

(55) Li, R.; Yang, J.; Han, J.; Liu, J.; Huang, M. Quantitative Determination of Melamine in Milk Using Ag Nanoparticle Monolayer Film as SERS Substrate. Phys. E Low-dimensional Syst. Nanostructures 2017, 88, 164-168. https://doi.org/10.1016/J.PHYSE.2016.12.013.

(56) Yu, W. W.; White, I. M. Chromatographic Separation and Detection of Target Analytes from Complex Samples Using Inkjet Printed SERS Substrates. Analyst 2013, 138 (13), 3679. https://doi.org/10.1039/c3an00673e.

(57) Durucan, O.; Rindzevicius, T.; Schmidt, M. S.; Matteucci, M.; Boisen, A. Nanopillar Filters for Surface-Enhanced Raman Spectroscopy. ACS Sensors 2017, 2 (10), 1400-1404. 
https://doi.org/10.1021/acssensors.7b00499.

(58) Yang, J.-L.; Yang, Z.-W.; Zhang, Y.-J.; Ren, H.; Zhang, H.; Xu, Q.-C.; Panneerselvam, R.; Sivashanmugan, K.; Li, J.-F.; Tian, Z.-Q. Quantitative Detection Using Two-Dimension ShellIsolated Nanoparticle Film. J. Raman Spectrosc. 2017, 48 (7), 919-924. https://doi.org/10.1002/jrs.5151.

(59) Sharma, Bhavya; Cardinal, M. Fernanda; Kleinman, Samuel L.; Greeneltch, Nathan G.; Frontiera, Renee R.; Blaber, Martin G.; Schatz, George C.; Van Duyne, R. P. HighPerformance SERS Substrates: Advances and Challenges. MRS Bull. 2013, 38 (8), 615-624. https://doi.org/10.1557/mrs.2013.161.

(60) Rajapandiyan, P.; Tang, W. L.; Yang, J. Rapid Detection of Melamine in Milk Liquid and Powder by Surface-Enhanced Raman Scattering Substrate Array. Food Control 2015, 56, 155 160. https://doi.org/10.1016/j.foodcont.2015.03.028.

(61) Kim, A.; Barcelo, S. J.; Williams, R. S.; Li, Z. Melamine Sensing in Milk Products by Using Surface Enhanced Raman Scattering. Anal. Chem. 2012, 84 (21), 9303-9309. https://doi.org/10.1021/ac302025q.

(62) Schmidt, M. S. S.; Hübner, J.; Boisen, A. Large Area Fabrication of Leaning Silicon Nanopillars for Surface Enhanced Raman Spectroscopy. Adv. Mater. 2012, 24 (10), OP11--8. https://doi.org/10.1002/adma.201103496.

(63) Skinner, C. G.; Thomas, J. D.; Osterloh, J. D. Melamine Toxicity. J. Med. Toxicol. 2010, 6 (1), 50-55. https://doi.org/10.1007/s13181-010-0038-1.

(64) $\mathrm{WHO} \mid$ International Experts Limit Melamine Levels in Food. WHO 2010. 
(65) He, H.; Sun, D. W.; Pu, H.; Chen, L.; Lin, L. Applications of Raman Spectroscopic Techniques for Quality and Safety Evaluation of Milk: A Review of Recent Developments. Crit. Rev. Food Sci. Nutr. 2019, 59 (5), 770-793. https://doi.org/10.1080/10408398.2018.1528436.

(66) Koglin, E.; Kip, B. J.; Meier, R. J. Adsorption and Displacement of Melamine at the Ag/Electrolyte Interface Probed by Surface-Enhanced Raman Microprobe Spectroscopy. J. Phys. Chem. 1996, 100 (12), 5078-5089. https://doi.org/10.1021/jp953208t.

(67) Bindesri, S. D.; Alhatab, D. S.; Brosseau, C. L. Development of an Electrochemical SurfaceEnhanced Raman Spectroscopy (EC-SERS) Fabric-Based Plasmonic Sensor for Point-of-Care Diagnostics. Analyst 2018, 143 (17), 4128-4135. https://doi.org/10.1039/c8an01117f.

(68) Analytical Method Development and Validation; 2018. https://doi.org/10.1201/9781315275161.

(69) Morelli, L.; Zór, K.; Jendresen, C. B.; Rindzevicius, T.; Schmidt, M. S.; Nielsen, A. T.; Boisen, A. Surface Enhanced Raman Scattering for Quantification of P-Coumaric Acid Produced by Escherichia Coli. Anal. Chem. 2017, $89 \quad$ (7), 3981-3987. https://doi.org/10.1021/acs.analchem.6b04428.

(70) Varnam, A. H.; Sutherland, J. P. Milk and Milk Products: Technology, Chemistry and Microbiology; Aspen Publishers, 2001.

(71) Robinson, A. M.; Harroun, S. G.; Bergman, J.; Brosseau, C. L. Portable Electrochemical Surface-Enhanced Raman Spectroscopy System for Routine Spectroelectrochemical Analysis. Anal. Chem. 2012, 84 (3), 1760-1764. https://doi.org/10.1021/ac2030078.

(72) Liu, B.; Lin, M.; Li, H. Potential of SERS for Rapid Detection of Melamine and Cyanuric Acid Extracted from Milk. Sens. Instrum. Food Qual. Saf. 2010, 4 (1), 13-19. 
https://doi.org/10.1007/s11694-009-9091-3.

\section{(73) PalmSens.PalmSens4}

https://www.palmsens.com/product/palmsens4/?gclid=Cj0KCQjw2K3rBRDiARIsAOFSW 6Oe-KD9zGweMkpkY23XgNiG1j90LfJnYwx50nlQS_7aN-

qX9fnNTQaAnjJEALw_wcB\#description (accessed Aug 31, 2019).

(74) Rajendran, S. T.; Scarano, E.; Bergkamp, M. H.; Capria, A. M.; Cheng, C.-H.; Sanger, K.; Ferrari, G.; Nielsen, L. H.; Hwu, E.-T.; Zór, K.; et al. Modular, Lightweight, Wireless Potentiostat-on-a-Disc for Electrochemical Detection in Centrifugal Microfluidics. Anal. Chem. 2019. https://doi.org/10.1021/acs.analchem.9b02026. 


\section{Graphical Abstract}

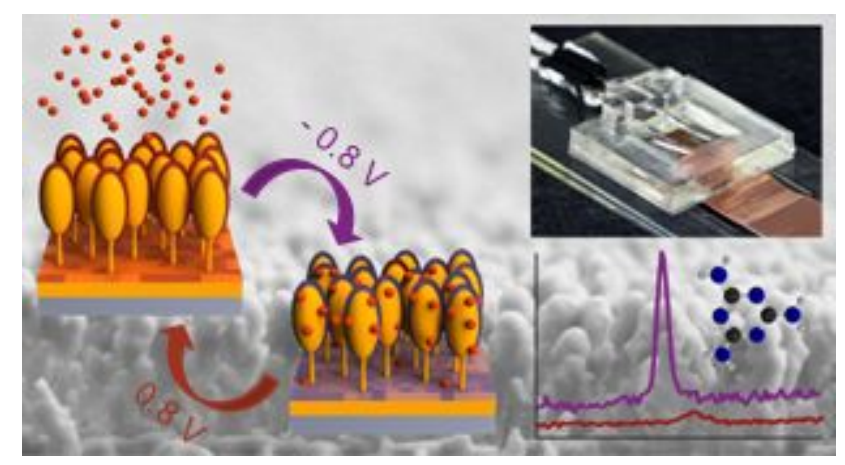

